\title{
Invasive meningococcal disease in patients with complement deficiencies: a case series (2008-2017)
}

\author{
Shamez N. Ladhani ${ }^{1,2^{*}}$ D, Helen Campbell ${ }^{1}$, Jay Lucidarme ${ }^{3}$, Steve Gray ${ }^{3}$, Sydel Parikh', Laura Willerton ${ }^{3}$, \\ Stephen A. Clark ${ }^{3}$, Aiswarya Lekshmi ${ }^{3}$, Andrew Walker ${ }^{3}$, Sima Patel $^{3}$, Xilian Bai ${ }^{3}$, Mary Ramsay ${ }^{1}$ and Ray Borrow ${ }^{3}$
}

\begin{abstract}
Background: To describe patients with inherited and acquired complement deficiency who developed invasive meningococcal disease (IMD) in England over the last decade.

Methods: Public Health England conducts enhanced surveillance of IMD in England. We retrospectively identified patients with complement deficiency who developed IMD in England during 2008-2017 and retrieved information on their clinical presentation, vaccination status, medication history, recurrence of infection and outcomes, as well as characteristics of the infecting meningococcal strain.

Results: A total of 16 patients with 20 IMD episodes were identified, including four with two episodes. Six patients had inherited complement deficiencies, two had immune-mediated conditions associated with complement deficiency (glomerulonephritis and vasculitis), and eight others were on Eculizumab therapy, five for paroxysmal nocturnal haemoglobinuria and three for atypical haemolytic uraemic syndrome. Cultures were available for 7 of 11 episodes among those with inherited complement deficiencies/immune-mediated conditions and the predominant capsular group was $Y(7 / 11)$, followed by $B(3 / 11)$ and non-groupable (1/11) strains. Among patients receiving Eculizumab therapy, 3 of the 9 episodes were due to group B (3/9), three others were NG but genotypically group $B$, and one case each of groups $E, W$ and $Y$.

Conclusions: In England, complement deficiency is rare among IMD cases and includes inherited disorders of the late complement pathway, immune-mediated disorders associated with low complement levels and patients on Eculizumab therapy. IMD due to capsular group Y predominates in patient with inherited complement deficiency, whilst those on Eculizumab therapy develop IMD due to more diverse capsular groups including non-encapsulated strains.
\end{abstract}

Keywords: Invasive meningococcal disease, Complement deficiency, Risk factors, Eculizumab

\section{Background}

Neisseria meningitidis (the meningococcus) remains a leading cause of bacterial meningitis and septicaemia worldwide, despite continued advances in the understanding of the pathogenesis of infection and development of new vaccines against this devastating infection. The meningococcus is commonly carried in the human

\footnotetext{
*Correspondence: shamez.ladhani@phe.gov.uk

'Immunisation and Countermeasures Division, Public Health England, 61 Colindale Avenue, London NW9 5EQ, UK

${ }^{2}$ Paediatric Infectious Diseases Research Group \& Vaccine Institute, Institute of Infection \& Immunity, St. Georges, University of London, London, UK Full list of author information is available at the end of the article
}

nasopharynx, especially in adolescents and young adults $[1,2]$. In recent years, genome-wide association studies [3] have identified host factors which may contribute to disease susceptibility; in particular, interaction between the complement system and the meningococcus has proven to be important in the pathogenesis of invasive meningococcal disease (IMD). IMD usually affects healthy individuals, in which a functional complement system acts as a first-line innate immune defence against invading pathogens [4]. Defects in components of the alternative pathway (properdin and factor D) as well as the terminal pathway (C5 to $\mathrm{C} 9$ ) underlie susceptibility

(c) The Author(s). 2019 Open Access This article is distributed under the terms of the Creative Commons Attribution 4.0 International License (http://creativecommons.org/licenses/by/4.0/), which permits unrestricted use, distribution, and reproduction in any medium, provided you give appropriate credit to the original author(s) and the source, provide a link to the Creative Commons license, and indicate if changes were made. The Creative Commons Public Domain Dedication waiver (http://creativecommons.org/publicdomain/zero/1.0/) applies to the data made available in this article, unless otherwise stated. 
to IMD [5]. Individuals with primary immunodeficiencies such as the autosomal recessive terminal complement pathway deficiencies have a 7000-10,000 fold higher risk of IMD compared to the general population and more than half of these patients develop recurrent episodes of IMD [6].

In addition to inherited deficiencies of the terminal complement pathway, a number of medical conditions and treatments can lead to acquired or secondary complement deficiency. In particular, Eculizumab (Soliris; Alexion) is a humanised monoclonal antibody that is a terminal complement pathway inhibitor used to treat paroxysmal nocturnal haemoglobinuria (PNH) [7] and atypical haemolytic uraemic syndrome (aHUS) [8], and its use is extending to treat other immune-mediated conditions [9-13]. Eculizumab binds with high affinity to human complement $\mathrm{C} 5$ and blocks the generation of C5a and C5b-9, which prevents the formation of membrane attack complexes and activation of the pro-inflammatory pathway, thus protecting against end-organ damage [7].

Characterising cases of IMD in individuals with complement deficiencies is fundamental to understanding disease risk in this highly vulnerable population and developing evidence-based guidance to both prevent and rapidly treat this potentially fatal condition. Public Health England (PHE) conducts enhanced national surveillance of IMD in England and routinely follows-up all cases confirmed by its national Meningococcal Reference Unit (MRU). Here we describe age distribution, clinical presentation, risk of recurrence, meningococcal typing and outcome of IMD in individuals with inherited or acquired complement deficiency diagnosed in England over a ten-year period.

\section{Methods}

In England, National Health Service (NHS) hospital laboratories routinely submit invasive meningococcal isolates to the PHE MRU for confirmation, grouping and additional characterisation [14]. The MRU also offers a free national PCR-testing service for patients with suspected IMD across England. IMD was defined as Neisseria meningitidis identified by culture or PCR from a sterile site. Confirmed cases are routinely followed up through postal questionnaires sent to their general practitioners for information on vaccination status, underlying co-morbidities, clinical presentation and outcomes of infection. For this study, IMD cases in patients with inherited or acquired complement deficiency diagnosed in England during 2008-2017 were included. Culture and non-culture samples submitted to the PHE MRU were characterised as reported previously [14-16].

\section{Results}

A total of 16 complement-deficient patients with 20 episodes of IMD were identified during the surveillance period (Table 1). For all 20 episodes, the diagnosis was confirmed by blood culture, PCR-positive whole blood EDTA or both. Of the four cases with two IMD episodes each, the first episode occurred prior to the surveillance period in two and, for the other two, both cases occurred during the surveillance period. In three of the four patients with repeat IMD episodes, the infections were due to different capsular groups; interestingly, all three involved one episode each of group B and group W IMD. In the fourth patient, the two IMD episodes occurred more than 2 years apart and were caused by a NG(B) strain (i.e. non-groupable because of interruption of ctrA gene but genotypically capsular group B) that was confirmed by PCR only; the $2 \mathrm{NG(B)}$ strains were indistinguishable in terms of PorA (P1.7-1,1) and fHbp (variant 2 peptide 23).

Six patients had inherited complement deficiencies, and two others had immune-mediated conditions associated with complement deficiency. The age range for those with inherited complement deficiencies or immune-mediated conditions was 4 to 42 years with a median age of 15 years. The first case with an immune-mediated condition affecting complement had hypocomplementaemic urticarial vasculitis (anti-C1q vasculitis) which was first diagnosed in 2010 when the patient developed infective endocarditis needing mitral valve repair, ischaemic cerebral events, lower limb amputation and endophthalmitis resulting in complete loss of vision in one eye. This patient developed IMD 3 years later whilst on immunosuppressive therapy for the underlying condition. The second case with an immune-mediated condition affecting complement developed IMD in 2012 and was diagnosed with membranoproliferative glomerulonephritis associated with persistently low complement factors C3 and C4 four months later.

Eight other patients were on Eculizumab therapy, five for paroxysmal nocturnal haemoglobinuria $(\mathrm{PNH})$ and three for atypical haemolytic uraemic syndrome (aHUS). The age range of patients on Eculizumab therapy was 20 to 40 years with a median age of 22 years. Overall, cultures were available for 7 of 11 episodes in patients with inherited complement deficiencies or immune-mediated conditions, and 6 of 9 episodes in patients receiving Eculizumab therapy despite national recommendations for daily antibiotic chemoprophylaxis in this vulnerable group. The rest of IMD episodes were confirmed by PCR only.

The predominant capsular group for the inherited complement deficiencies or immune-mediated conditions was capsular group Y (7/11), followed by capsular group B (3/11); one strain that was non-groupable by PCR (i.e. ctrA positive but not positive for capsular 


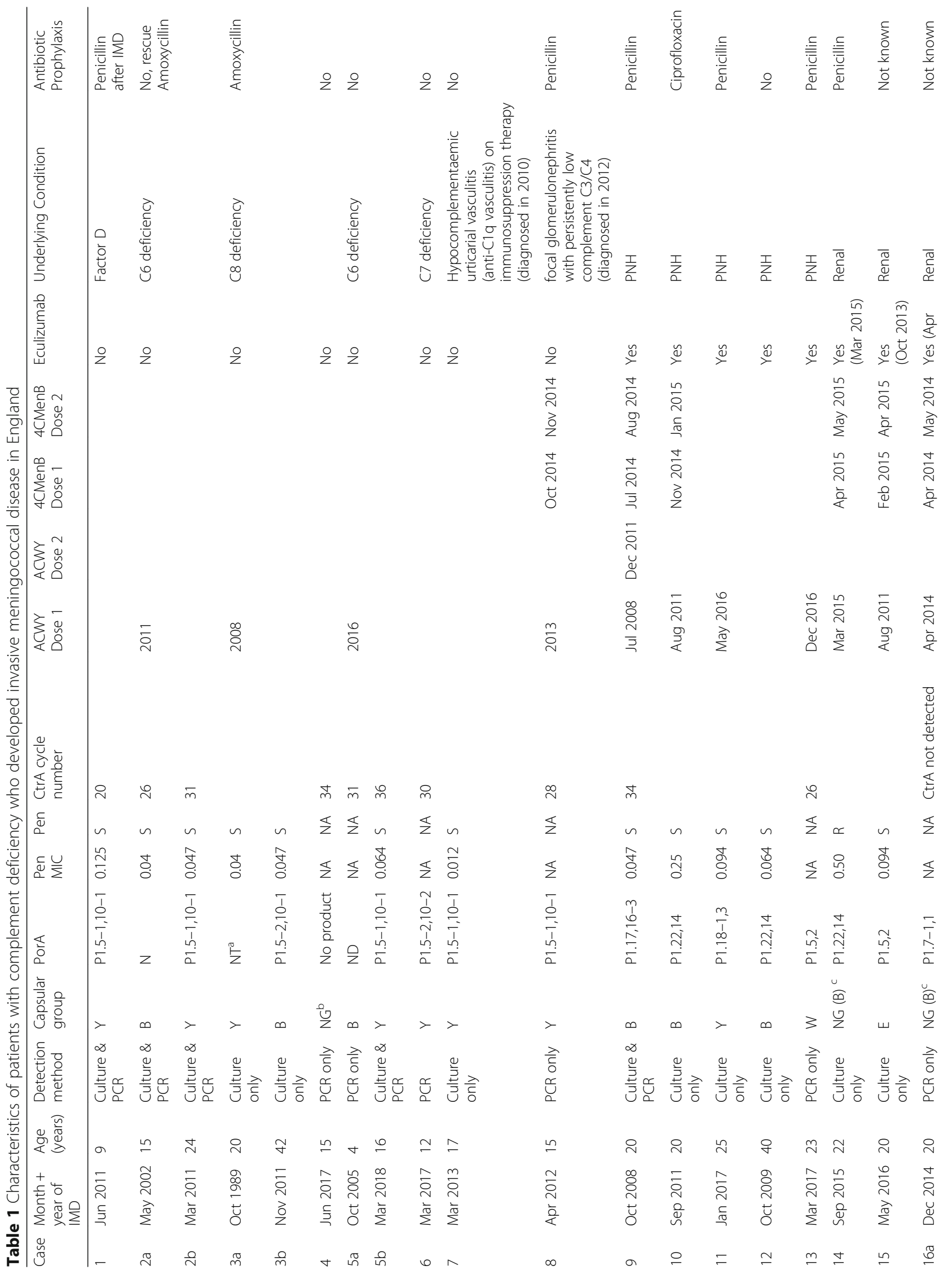




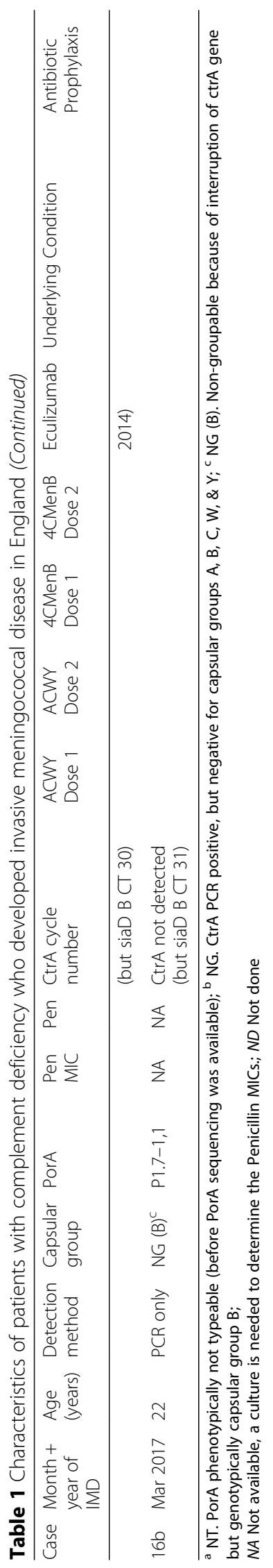


groups A, B, C, W or Y), suggesting a truly nongroupable strain or a rare capsular group. For those on Eculizumab therapy, six of the nine isolates were due to either capsular group B $(n=3)$ or non-groupable (interruption of ctrA gene) but genotypically capsular group $\mathrm{B}$ (NG(B); $\mathrm{n}=3$ ). Three other episodes were due to capsular groups W, Y and E. Capsular group Y strains was mainly associated with PorA P1.5-1,10-1. One isolate was penicillin-resistant - a non-groupable strain that was genotypically characterised as group B [17].

Four cases had recurrent IMD episodes, three who were diagnosed with an inherited complement deficiency after their second IMD episode, and one in a renal patient on Eculizumab therapy. Case 2 with complement C6 deficiency developed IMD due to capsular group B at 15 years of age, followed by IMD due to capsular group Y 9 years later. This case was on rescue antibiotics (i.e. self-treatment with a treatment course of amoxycillin when unwell) at the time and only received the MenACWY conjugate vaccine after the second episode of IMD. Case 3 with complement C8 deficiency had two episodes of IMD at 20 and 42 years of age due to capsular group $\mathrm{Y}$ and $\mathrm{B}$, respectively; this patient was prescribed prophylactic amoxycillin twice daily following the second episode. Case 5 was diagnosed with complement $\mathrm{C} 6$ deficiency after the second IMD episode. This case developed IMD due to capsular group B at 4 years of age and then with capsular group $Y$ at 16 years; the patient had received the MenACWY conjugate vaccine as part of the national UK adolescent programme 2 years prior to the second episode of IMD. Case 16 with aHUS commenced Eculizumab therapy aged 20 years and presented with IMD at 20 and 22 years of age after been appropriately vaccinated with a single dose of MenACWY conjugate vaccine and two doses of the meningococcal group B vaccine, 4CMenB. This patient was reported to be intermittently non-compliant with antibiotics. Both IMD episodes were confirmed by PCR only; the infecting strains were both PCR negative for the ctrA gene, but genotypically confirmed as capsular group B. Both strains had porA P1.7-1,1 and factor $\mathrm{H}$ binding protein variant 2 allele 23 peptide 23 . Neither of these antigenic variants are predicted to be covered by 4CMenB.

\section{Discussion}

We identified 16 individuals with invasive meningococcal disease who had confirmed primary or acquired complement deficiency in England over a decade. Most cases developed IMD during adolescence and early adulthood, including four who developed recurrent IMD; three of the four cases were diagnosed with an underlying primary complement deficiency after the second IMD episode. In addition to the primary complement deficiencies, we identified two groups with acquired complement deficiency, those with immune disorders leading to secondary complement deficiency and iatrogenic cases in patients receiving Eculizumab, a humanised monoclonal complement C5 inhibitor.

The complement system is an important component of the innate immune defence against invading bacteria including meningococci; complement activation on cell surfaces may be initiated through any of the three pathways - the classical, lectin, or alternative pathways. The terminal complement pathway comprises five proteins (C5-C9) that combine together to form a lethal pore-like membrane attack complex (MAC), which disrupts the bacterial cell membrane and forms transmembrane channels, leading to bacterial lysis [4]. Complement deficiencies represent approximately $1-6 \%$ of all primary immunodeficiencies but this may go up to $10 \%$ of primary immunodeficiencies in certain communities [18]. The prevalence of inherited complement deficiency has been calculated to be about $0.03 \%$ in the general European population [18], excluding mannose-binding lectin (MBL) deficiency, which has been estimated to occur in its homozygous form in about $5 \%$ of the population [19].

In our cohort, we observed an over-representation of IMD due to capsular group $\mathrm{Y}$ in those with inherited complement deficiencies $(7 / 11,64 \%)$ compared to those receiving Eculizumab therapy $(1 / 9,11 \%)$; the risk of group Y IMD in the latter group is similar to the rest of the population - in England, IMD due to group Y was responsible for $10.9 \%$ of all cases during 2011-15 [20]. In the UK, group $\mathrm{Y}$ is commonly carried in teenagers, with nasopharyngeal carriage rates of $10.0 \%, 10.1 \%$ and 9.7\% among 15-19 year olds during 1999, 2000 and 2001, respectively [21]. During 2010-11, carriage of group Y meningococci among 18-24 year-olds in England was $7 \%[22]$.

Another significant difference between the inherited complement deficiencies and the patients on Eculizumab therapy was that all but one strain (8/9 strains, including six with group $\mathrm{Y}$ and two with group B) causing IMD in patients with inherited complement deficiencies were encapsulated, as were both the strains causing IMD in patients with immune-mediated conditions associated with complement deficiency (both group Y). In contrast, four of the nine IMD episodes in patients on Eculizumab therapy were either NG or group E; these strains are less virulent and usually only associated with carriage.

Our findings support the recent French report of 56 cases with terminal complement pathway deficiency who developed 61 episodes IMD between 1980 and 2015 [5]. They also found that patients with terminal complement deficiency were more likely to develop IMD due to minor or uncommon meningococcal serogroups, especially MenY 
which was responsible for 44\% (27/61) of all cases, but also W and NG strains, although no correlation was found between specific terminal complement deficiencies and capsular group or clonal complex. Notably, as in our study, most isolates from patients with terminal complement deficiency were groupable, indicating that they were able to express capsule and could, therefore, be pathogenic [5].

A new and significant finding in our cohort was that there were more patients with IMD who were on Eculizumab treatment than those with primary terminal complement deficiency, including six treated for $\mathrm{PNH}$ and three for aHUS. An unwanted complication of complement inhibition, however, is an increased risk of infection with encapsulated bacteria, especially IMD. Unfortunately, just like patients with primary terminal complement deficiency, this group remains at increased risk of IMD even after appropriate immunisation because they are unable to mediate meningococcal lysis by the membrane attack complex. This finding emphasises the importance of penicillin chemoprophylaxis to prevent IMD and other serious bacterial infections in high-risk patients. Whilst there are no published guidelines on the management of individuals with inherited complement deficiencies or immune-mediated conditions associated with hypocomplementaemia, antibiotic chemoprophylaxis is routinely recommended for those with asplenia and those receiving Eculizumab. We have, however, recently reported a case of penicillin-resistant MenB disease in a vaccinated young adult on penicillin prophylaxis, highlighting the need to raise awareness of IMD risk among patients and healthcare professionals, as well as the need to seek early medical attention and for early investigation and treatment in unwell patients receiving Eculizumab, who usually present with nonspecific symptoms and signs of infection. Although none of the patients in our cohort died of IMD, there have been reports of fatalities due to NG strains in patients receiving Eculizumab [23].

In addition to the known risk factors for IMD, we found other rare conditions associated with complement deficiencies, such as hypocomplementaemic urticarial vasculitis (anti-C1q vasculitis), which is associated with glomerulonephritis [24] as well as some forms of membranoproliferative glomerulonephritis. Conditions associated with acquired immune deficiencies include inadequate complement production (e.g., severe liver dysfunction), increased complement consumption (autoimmune disorders, diseases associated with immune complex formation), or increased excretion of complement components (e.g., protein-losing nephropathies) [4]. Around half the patients with systemic lupus erythematosus (SLE) have low C3 and C4 complement concentrations. C3 is the most abundant complement protein and its cleavage and the stable, covalent linkage of its fragments to target surfaces is a critical outcome of complement activation. Notably, some forms of glomerulonephritis, such as those associated with C3 nephritic factor, have responded to treatment with Eculizumab [25].

IMD in patients with complement deficiency is generally associated with mild disease, possibly due to a less intense inflammatory response in the absence of an intact complement pathway. Fatal cases have, however, been reported, including IMD cases due to NG meningococci, which generally only attack vulnerable hosts [23]. In addition to highly effective polysaccharide-conjugate vaccines against group A, C, W and Y meningococcal disease (MenACWY), there are two new broad-spectrum, protein-based meningococcal vaccines licensed in Europe. These vaccines are highly immunogenic in children and adults, and $4 \mathrm{CMenB}$ has been shown to protect against IMD in infants and toddlers in the field [26]. Patients with complement deficiency, however, may still develop IMD despite high postimmunisation antibody titres, again because of the lack of a functional complement pathway; vaccine failure after both MenACWY and 4 CMenB have been reported in this vulnerable group, leading some specialists to recommend daily penicillin prophylaxis.

\section{Conclusions}

Acquired and inherited deficiencies of the terminal complement pathway are rare, even in patients with IMD. Most cases of IMD in patients with inherited complement deficiency occur during adolescence. Testing for complement deficiency after a single episode of IMD is not routinely recommended because the vast majority of patients do not have any underlying co-morbidities. Healthcare professionals should, however, consider the possibility of inherited complement deficiency in any patient with recurrent IMD or in those who develop IMD due to unusual capsular groups such as Y, E or NG meningococci, especially in previously healthy adolescents. The management of such patients is challenging because of the limited protection offered by the current vaccines. In addition to vaccination, antibiotic chemoprophylaxis should be strongly considered but rare cases of penicillin-resistant group B meningococcal disease highlight the need to raise awareness of IMD risk among patients and healthcare professionals, including use of information cards to be carried by patients and their care givers, as well as the need to seek early medical attention and for early investigation and treatment in unwell patients.

\section{Abbreviations}

aHUS : Atypical haemolytic uraemic syndrome; IMD: Invasive meningococcal disease; MenACWY: Meningococcal group A, C, W and Y;

MenB: Meningococcal group B; PNH: Paroxysmal nocturnal haemoglobinuria

\section{Acknowledgements}

Angela Watt and Joanne Stout, Nurse Specialists, National aHUS Service, Building 26 Royal Victoria Infirmary, Queen Victoria Road, Newcastle upon 
Tyne, NE1 4LP, UK. Louise Arnold, Clinical Nurse Specialist, PNH Team, St James's hospital, Leeds, UK. Drs Peter Arkwright and Stephen Hughes, Consultant Paediatric Allergist and Immunologist, Royal Manchester Children's Hospital, Manchester, M13 9WP, UK.

\section{Authors' contributions}

RB developed the idea; SNL and MR are the clinical leads for meningococcal disease surveillance in England; $\mathrm{HC}$ and SP conduct the national surveillance for meningococcal disease; AL, JL, SG, LW, SC, AW, SP, XB and RB conduct laboratory surveillance for IMD; SP wrote the first draft of the manuscript; al authors contributed to the interpretation of the results and discussion; all authors approved the final submitted version of the manuscript.

\section{Funding}

None.

\section{Availability of data and materials}

All data generated or analysed during this study are included in this published article [and its supplementary information files].

\section{Ethics approval and consent to participate}

PHE has legal permission, provided by Regulation 3 of The Health Service (Control of Patient Information) Regulations 2002, to process patient confidential information for national surveillance of communicable diseases (http://www.legislation.gov.uk/uksi/2002/1438/regulation/3/made). This includes PHE's responsibility to monitor the safety and effectiveness of vaccines. Individual patient consent is, therefore, not required by PHE from IMD cases.

\section{Consent for publication}

Not applicable.

\section{Competing interests}

AL, AW, JL, LW, RB, SAC, SG, SL, SP and XB perform contract research on behalf of Public Health England for GSK, Pfizer and Sanofi Pasteur. All other authors: no conflict.

\section{Author details}

${ }^{1}$ Immunisation and Countermeasures Division, Public Health England, 61 Colindale Avenue, London NW9 5EQ, UK. ${ }^{2}$ Paediatric Infectious Diseases Research Group \& Vaccine Institute, Institute of Infection \& Immunity, St Georges, University of London, London, UK. ${ }^{3}$ Meningococcal Reference Unit, Public Health England, Manchester Royal Infirmary, Manchester, UK.

\section{Received: 23 January 2019 Accepted: 30 May 2019}

\section{Published online: 14 June 2019}

\section{References}

1. Christensen H, May M, Bowen L, Hickman M, Trotter CL. Meningococcal carriage by age: a systematic review and meta-analysis. Lancet Infect Dis. 2010;10:853-61.

2. Caugant DA, Maiden MC. Meningococcal carriage and disease--population biology and evolution. Vaccine. 2009:27(Suppl 2):B64-70.

3. Davila S, Wright VJ, Khor CC, Sim KS, Binder A, Breunis WB, et al. Genomewide association study identifies variants in the CFH region associated with host susceptibility to meningococcal disease. Nat Genet. 2010;42:772-6.

4. Lewis LA, Ram S. Meningococcal disease and the complement system. Virulence. 2014;5:98-126.

5. Rosain J, Hong E, Fieschi C, Martins PV, El Sissy C, Deghmane AE, et al. Strains responsible for invasive meningococcal disease in patients with terminal complement pathway deficiencies. J Infect Dis. 2017:215:1331-8.

6. Figueroa J, Andreoni J, Densen P. Complement deficiency states and meningococcal disease. Immunol Res. 1993;12:295-311.

7. Hillmen P, Young NS, Schubert J, Brodsky RA, Socié G, Muus P, et al. The complement inhibitor eculizumab in paroxysmal nocturnal hemoglobinuria. N Engl J Med. 2006:355:1233-43.

8. Legendre CM, Licht C, Muus P, Greenbaum LA, Babu S, Bedrosian C, et al. Terminal complement inhibitor eculizumab in atypical hemolytic-uremic syndrome. N Engl J Med. 2013;368:2169-81.
9. Reis ES, Mastellos DC, Yancopoulou D, Risitano AM, Ricklin D, Lambris JD. Applying complement therapeutics to rare diseases. Clin Immunol. 2015; 161:225-40

10. Nester CM, Brophy PD. Eculizumab in the treatment of atypical haemolytic uraemic syndrome and other complement-mediated renal diseases. Curr Opin Pediatr. 2013;25:225-31.

11. Bomback AS. Eculizumab in the treatment of membranoproliferative glomerulonephritis. Nephron Clin Pract. 2014;128:270-6.

12. Misawa S, Kuwabara S, Sato Y, Yamaguchi N, Nagashima K, Katayama K, et al. Safety and efficacy of eculizumab in Guillain-Barré syndrome: a multicentre, double-blind, randomised phase 2 trial. Lancet Neurol. 2018;17: 519-29.

13. Paul F, Murphy O, Pardo S, Levy M. Investigational drugs in development to prevent neuromyelitis optica relapses. Expert Opin Investig Drugs. 2018:27:265-71.

14. Gray SJ, Trotter CL, Ramsay ME, Guiver M, Fox AJ, Borrow R, et al. The epidemiology of meningococcal disease in England and Wales 1993/94 to 2003/04: the contribution and experiences of the meningococcal reference unit. J Med Microbiol. 2006;55:887-96.

15. Chatt C, Gajraj R, Hawker J, Neal K, Tahir M, Lawrence M, et al. Four-month outbreak of invasive meningococcal disease caused by a rare serogroup $B$ strain, identified through the use of molecular PorA subtyping, England, 2013. Euro Surveill. 2014;19(44). https://www.eurosurveillance.org/content/ 10.2807/1560-7917.ES2014.19.44.20949.

16. Hill DM, Lucidarme J, Gray SJ, Newbold LS, Ure R, Brehony C, et al. Genomic epidemiology of age-associated meningococcal lineages in national surveillance: an observational cohort study. Lancet Infect Dis. 2015;15:1420-8.

17. Parikh SR, Lucidarme J, Bingham C, Warwicker P, Goodship T, Ramsay ME, Borrow R, Ladhani SN. First report of meningococcal B vaccine failure in a young adult on long-1 term eculizumab. Pediatrics. 2017;140. https:// pediatrics.aappublications.org/content/140/3/e20162452.long

18. Grumach AS, Kirschfink M. Are complement deficiencies really rare? Overview on prevalence, clinical importance and modern diagnostic approach. Molecular Immunol. 2014;61:110-7.

19. Dahl M, Tybjaerg-Hansen A, Schnohr P, Nordestgaard BG. A population based study of morbidity and mortality in mannose binding lectin deficiency. J Exp Med. 2004:199:1391-9.

20. Parikh SR, Campbell H, Gray SJ, Beebeejaun K, Ribeiro S, Borrow R, Ramsay ME, Ladhani SN. Epidemiology, clinical presentation, risk factors, intensive care admission and outcomes of invasive meningococcal disease in England, 2010-2015. Vaccine. 2018;36:3876-81.

21. Maiden MC, Ibarz-Pavón AB, Urwin R, Gray SJ, Andrews NJ, Clarke SC, et al. Impact of meningococcal serogroup $C$ conjugate vaccines on carriage and herd immunity. J Infect Dis. 2008;197:737-43.

22. Read RC, Baxter D, Chadwick DR, Faust SN, Finn A, Gordon SB, et al. Effect of a quadrivalent meningococcal ACWY glycoconjugate or a serogroup $B$ meningococcal vaccine on meningococcal carriage: an observer-blind, phase 3 randomised clinical trial. Lancet. 2014;384:2123-31.

23. Nolfi-Donegan D, Konar M, Vianzon V, MacNeil J, Cooper J, Lurie P, et al. Fatal Nongroupable Neisseria meningitidis disease in vaccinated patient receiving Eculizumab. Emerg Infect Dis. 2018;24(8). https://doi.org/10.3201/ eid2408.180228.

24. Sjöwall C, Mandl T, Skattum L, Olsson M, Mohammad AJ. Epidemiology of hypocomplementaemic urticarial vasculitis (anti-C1q vasculitis). Rheumatology (Oxford). 2018:57:1400-7.

25. Garg N, Zhang Y, Nicholson-Weller A, Khankin EV, Borsa NG, Meyer NC, et al. $\mathrm{C} 3$ glomerulonephritis secondary to mutations in factors $\mathrm{H}$ and $\mathrm{l}$ : rapid recurrence in deceased donor kidney transplant effectively treated with eculizumab. Nephrol Dial Transplant. 2018. https://doi.org/10.1093/ndt/ gfx369 [Epub ahead of print].

26. Parikh SR, Andrews NJ, Beebeejaun K, Campbell H, Ribeiro S, Ward C, et al. Effectiveness and impact of a reduced infant schedule of $4 C M e n B$ vaccine against group B meningococcal disease in England: a national observational cohort study. Lancet. 2016;388:2775-82.

\section{Publisher's Note}

Springer Nature remains neutral with regard to jurisdictional claims in published maps and institutional affiliations. 